\title{
An adaptive operation strategy for voltage stability enhancement in DMFCs
}

\author{
Qin-Wen YANG ${ }^{a}$, Xu-Qu HU', ${ }^{a}$, Ying ZHU ${ }^{a}$, Xiu-Cheng LEI ${ }^{a}$, Xing-Yi \\ $\mathrm{WANG}^{\mathrm{a}}$ \\ ${ }^{a}$ State Key Laboratory of Advanced Design and Manufacturing for Vehicle Body, College of \\ Mechanical and Vehicle Engineering, Hunan University, Changsha, 410082, China
}

\begin{abstract}
An adaptive operation strategy for on-demand control of DMFC system is proposed as an alternative method to enhance the voltage stability. Based on a single-cell DMFC stack, a newly simplified semi-empirical model is developed from the uniform-designed experimental results to describe the I-V relationship. Integrated with this model, the multi-objective optimization method is utilized to develop an adaptive operation strategy. Although the voltage instability is frequently encountered in unoptimized operations, the voltage deviation is successfully decreased to a required level by adaptive operations with operational adjustments. Moreover, the adaptive operations are also found to be able to extend the range of operating current density or to decrease the voltage deviation according to ones requirements. Numerical simulations are implemented to investigate the underlying mechanisms of the proposed adaptive operation strategy, and experimental adaptive operations are also performed on another DMFC system to validate the adaptive operation strategy. Preliminary experimental
\end{abstract}

\footnotetext{
${ }^{*}$ Corresponding author

Email address: huxuqu@gmail.com (Xu-Qu HU)
}

Preprint submitted to Preprints.org

January 10, 2018 
study shows a rapid response of DMFC system to the operational adjustment, which further validates the effectiveness and feasibility of the adaptive operation strategy in practical applications. The proposed strategy contributes to a guideline for the better control of output voltage from operating DMFC systems. Keywords: Direct Methanol Fuel Cell, Operation strategy, Multi-objective optimization

\section{Introduction}

Direct methanol fuel cell (DMFC) has emerged in recent years as a promising power source for various kinds of electronic devices [1, 2]. It can provide high energy density and almost instant recharging with simple system configurations [3]. But to move towards real-time operations of DMFCs that meet a commercially acceptable standard, further improvements are still needed especially in the fields of systematic stability. During the past decades, lots of research efforts have been dedicated to enhance the system stability in DMFCs by improving the catalyst loading [4, 5], the fuel cell structure [6, 7] and the fuel delivery system [3, 8], etc.

As the working current usually varies in a wide range, one problem that received relatively less attention is the voltage instability. Because of the polarization effect, the over-potential phenomenon is normally inevitable. An obvious voltage deviation is often encountered, even if the DMFC was assumed to be operated in the theoretical ohmic loss range [8, 9]. Many published experimental results have shown that the output voltage could be very sensitive to a small variation of current density [2, 5, 6, 7]. Although a voltage stabilizer can be 
useful to a certain extent, an operation strategy is in great need to avoid additional energy loss caused by the process of voltage stabilization, and it can be necessary while the voltage deviation is beyond the input limit of voltage stabilizers.

Several control strategies have been applied to analyze and enhance the system stability of DMFCs, ranging from PID control algorithms [10], neural network methods [11] to model predictive control schemes [12, 13], but most of them were designed to work with the assumption of small disturbance (or little noise). Since the operating condition in commercial electric equipments would change sharply, the influence of large disturbance on the voltage stability need to be taken into account. An adaptive operation strategy that is suitable for large disturbance in complex operating conditions becomes the initial motivation of the present study.

However, it is a task full of challenges, as it usually requires accurate models of DMFC systems which are necessary for the study of acting mechanisms of operating parameters. During the past decades, several mechanical models were proposed to study the energy conversion process of DMFCs, from onedimensional [14, 15], two-dimensional [16, 17, 18] to three-dimensional [19, 20, 21. point of view. With the consideration of large variations of operating parameters, we have proposed an effective semi-empirical model to describe I-V relationship and developed a three-dimensional Computational Fluid Dynamics (CFD) model to study the DMFC performance [22]. More recently, it has been successfully applied to determine the optimal operating conditions of a DMFC 
system [23]. Although the effects of operating conditions, such as temperature, methanol concentration and solution/air flow rates, on the DMFC performance

can be well estimated using those existing models [24, 25], an adaptive operation strategy that optimally controls the operating conditions can still be necessary for the enhancement of voltage stability in practical applications.

An adaptive operation strategy was developed to enhance the voltage stability in DMFC systems, and a systematic study about the performance and underlying mechanisms of this strategy was performed. In Section 2 , the procedure of strategy establishment and the details about the applied methods, ranging from the experimental set-up, semi-empirical model to the numerical techniques, were all presented. In Section 3 , the theoretical and experimental results were systematically provided to study the necessity, feasibility and superiority of the proposed strategy, and the numerical simulations were also performed to investigate the underlying mechanisms. Conclusions and perspectives were summarized in Section 4

\section{Methods and models}

The adaptive operation strategy was developed based on the integration of analytical, experimental and numerical techniques. Firstly, experimental study was performed on the platform of a single-cell DMFC stack. The I-V relationships of this stack were recorded as the main operating parameters were changed according to the uniform design principal. A simplified semi-empirical model was then proposed to quantitatively describe the DMFC performance through 
careful analysis on the experimental data. Based on the semi-empirical model, the adaptive operation strategy was developed using multi-objective optimization method. The numerical model that was used to investigate the underlying mechanism of the energy conversion process in adaptive DMFC operations was also presented.

\subsection{Experimental setup}

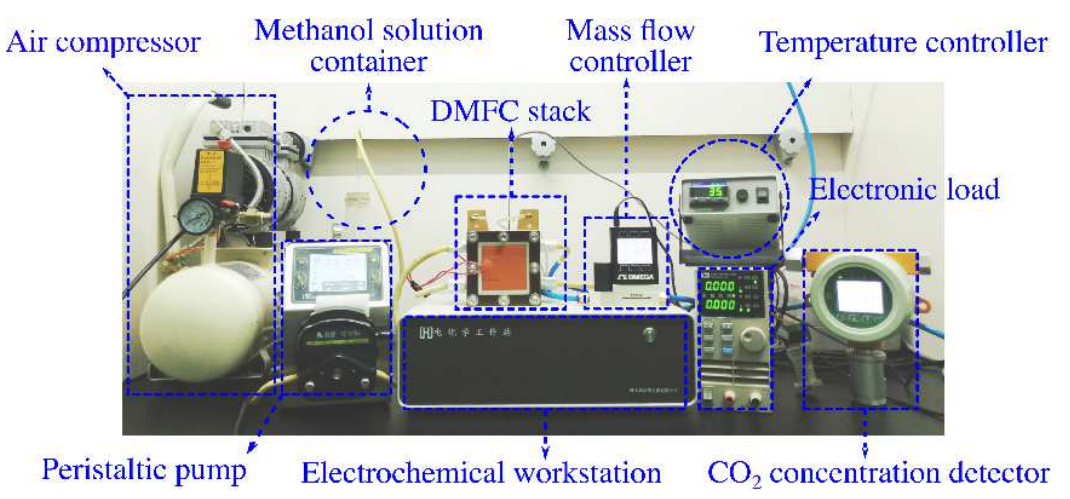

Figure 1: Experimental setup of the DMFC system.

Experimental set-up is shown in Fig. 1) A single-cell DMFC stack (TeskStak, Parker Hannifin Energy Systems) consists of a five-layer Membrane Electrode Assembly (MEA) sandwiched by two graphite end plates (for anode and cathode). Serpentine flow channel of 12 U-turns is integrated inside the end plates, which takes a rectangular cross-section $\left(30.94 \times 30.65 \mathrm{~mm}^{2}\right)$ and a total length of $415.06 \mathrm{~mm}$. At the anode side, the methanol solution composed by deionized water and pure methanol is flowed into the channel by a peristaltic pump (BT300LC). At the cathode side, an air compressor (OUTSTANDING OTS550) regulated by a rotameter (OMEGA FMA-2605A) is used to pump the air 
into the fuel cell. The working temperature of the DMFC stack is maintained by a supplementary heating apparatus that is controlled by a temperature controller (Omega CSC32). An electronic load device (ITECH it8211) is used to change the current density to different levels and to measure the corresponding values of the voltage. The DMFC performance is monitored by an electrochemical workstation (CHI660E), while the production of $\mathrm{CO}_{2}$ is measured by a $\mathrm{CO}_{2}$ concentration detector (JA500-CO2-IR1).

Table 1: Value ranges of main operating parameters.

\begin{tabular}{|c|c|c|c|}
\hline Name & Value range & Name & Value range \\
\hline Temperature (K) & {$[298,343]$} & Methanol concentration (M) & {$[0.25,2]$} \\
\hline Air flow rate $(\mathrm{ccm})$ & {$[81.2,186]$} & Feed solution flow rate $(\mathrm{ccm})$ & {$[3.5,5.5]$} \\
\hline
\end{tabular}

Four main operating parameters were considered in experimental study, including the working temperature $(T)$, the methanol concentration $\left(C_{M}\right)$, the input flow rates of feed methanol solution $\left(F_{M}\right)$ and the air $\left(F_{A}\right)$. The values of the operating parameters, which were selected based on literature review and practical experience, are summarized in Table 1, In order to consider the influence of operating parameters on the output voltage, a systematic experimental work was designed based on the principal of uniform design [26]. As five different levels of each operating parameter were selected, 65 experimental tests were performed for the four main operating parameters, 45 of which are decided by uniform design, and the other 20 ones are the supplementary test cases for the analysis of DMFC performance degradation. All the operating parameters for these uniform-designed experimental cases were summarized in Table 2. 
Table 2: Operating parameters for uniform-designed experiments.

\begin{tabular}{cccccccccc}
\hline No. & $T(\mathrm{~K})$ & $C_{M}(\mathrm{M})$ & $F_{M}(\mathrm{ccm})$ & $F_{A}(\mathrm{ccm})$ & $N_{o .}$ & $T(\mathrm{~K})$ & $C_{M}(\mathrm{M})$ & $F_{M}(\mathrm{ccm})$ & $F_{A}(\mathrm{ccm})$ \\
\hline 1 & 298 & 1 & 5.5 & 93.6 & 2 & 333 & 1 & 4.5 & 125.5 \\
3 & 323 & 2 & 4 & 81.2 & 4 & 333 & 0.25 & 3.5 & 81.2 \\
5 & 323 & 1.5 & 5 & 93.6 & 6 & 298 & 1.5 & 4 & 81.2 \\
7 & 323 & 0.5 & 4 & 125.2 & 8 & 323 & 0.5 & 4.5 & 125.2 \\
9 & 343 & 2 & 3.5 & 93.6 & 10 & 313 & 1 & 4 & 108.7 \\
11 & 298 & 0.5 & 4.5 & 125.2 & 12 & 343 & 1.5 & 4.5 & 81.2 \\
13 & 333 & 0.5 & 5.5 & 108.7 & 14 & 313 & 1.5 & 4.5 & 108.7 \\
15 & 323 & 1.5 & 5 & 125.2 & 16 & 323 & 0.25 & 5.5 & 93.6 \\
17 & 313 & 2 & 4.5 & 140.8 & 18 & 333 & 1.5 & 3.5 & 108.7 \\
19 & 313 & 1 & 3.5 & 81.2 & 20 & 323 & 1.5 & 3.5 & 140.8 \\
21 & 313 & 2 & 5.5 & 81.2 & 22 & 298 & 0.5 & 3.5 & 93.6 \\
23 & 313 & 0.25 & 3.5 & 108.7 & 24 & 298 & 1.5 & 5.5 & 140.8 \\
25 & 298 & 2 & 3.5 & 125.2 & 26 & 313 & 0.25 & 5.5 & 125.2 \\
27 & 333 & 1 & 5 & 81.2 & 28 & 313 & 0.5 & 4.5 & 93.6 \\
29 & 343 & 0.5 & 5.5 & 81.2 & 30 & 333 & 1 & 5.5 & 140.8 \\
31 & 343 & 0.25 & 5 & 140.8 & 32 & 333 & 2 & 4 & 140.8 \\
33 & 343 & 0.5 & 3.5 & 140.8 & 34 & 333 & 0.25 & 4.5 & 93.6 \\
35 & 343 & 1.5 & 5 & 108.7 & 36 & 298 & 0.25 & 5 & 81.2 \\
37 & 323 & 1 & 4 & 93.6 & 38 & 333 & 2 & 4.5 & 93.6 \\
39 & 298 & 0.25 & 4 & 140.8 & 40 & 343 & 0.25 & 4 & 108.7 \\
41 & 343 & 2 & 5.5 & 125.2 & 42 & 313 & 1 & 5 & 140.8 \\
43 & 298 & 2 & 5 & 108.7 & 44 & 323 & 0.5 & 5 & 108.7 \\
45 & 343 & 1 & 4 & 125.2 & 46 & 323 & 0.25 & 4.5 & 186 \\
47 & 323 & 0.5 & 4.5 & 186 & 48 & 323 & 1 & 4.5 & 186 \\
\hline & & & & & & & & & 5 \\
\end{tabular}

\subsection{Semi-empirical model}

Since the degradation of DMFC performance was observed in experiments, a linear regression method was applied in the collection process of experimental data [22, 27]. Careful investigation was implemented to analyze the compensated experimental data, and a semi-empirical model can be deduced to describe the relationship between the operating parameters and the output voltage.

A semi-empirical model was developed to quantify the influence of main operating parameters on the DMFC output in our previous study [21, 22], but it contains a large number of undetermined coefficients and the adaptation work 
usually involves a large amount of computations. Based on the electrochemical theory, a simplified semi-empirical model with less undetermined coefficients was proposed in the present study. It can be expressed as,

$$
\begin{aligned}
V_{\text {cell }} & =1.21+k_{1} T \ln \left(C_{M}\right)+k_{3} T \ln \left(F_{A}\right)+k_{4} \\
& -k_{5} e^{\left(k_{6} / T+k_{7}\right)} j-\left(k_{8} j^{2}+k_{9} T+k_{10} C_{M}^{2}+k_{11} C_{M}+k_{12}\right) \\
& \times\left[\ln (j)+k_{13}+k_{14} \ln \left(\frac{C_{M}}{1-\frac{j}{k_{15} e^{\left(-k_{16} / T\right)} C_{M}^{2}}}\right)\right] \\
& -\left(k_{17} j+k_{18} T+k_{19} C_{M}^{2}+k_{20} C_{M}+k_{21}\right) \\
& \times\left[\ln (j)+k_{22} \ln \left(F_{A}\right)+k_{23}\right]+k_{24} j^{2} \ln \left(F_{M}\right),
\end{aligned}
$$

where $V_{\text {cell }}$ is the output voltage from full cell, $j$ denotes the current density, and the operating parameters $T, C_{M}, F_{M}, F_{A}$ are defined as mentioned above. The 24 undetermined coefficients can be identified from those compensated experimental data using a non-linear regression method, which are $k_{1}=-3.7534, k_{2}=$ $-3.1534, k_{3}=6.6200, k_{4}=-0.7499, k_{5}=6.9897, k_{6}=916.91, k_{7}=-4.6392$, $k_{8}=-0.8801, k_{9}=-0.5791, k_{10}=-4.8053, k_{11}=4.8053, k_{12}=-1.2013$, $k_{13}=-36.4679, k_{14}=2.8579 \times 10^{-4}, k_{15}=5.34657 \times 10^{7}, k_{16}=5182.4$, $k_{17}=-0.1160, k_{18}=0.5793, k_{19}=4.8060, k_{20}=-4.8060, k_{21}=1.2015$, $k_{22}=-8.2583 \times 10^{-4}, k_{23}=-36.4679$ and $k_{24}=-29.8716$.

Fig. 2 shows the comparison between the semi-empirical predications and experimental data. The experimental relationship of current density and output voltage can be well estimated by the proposed semi-empirical model, even if 


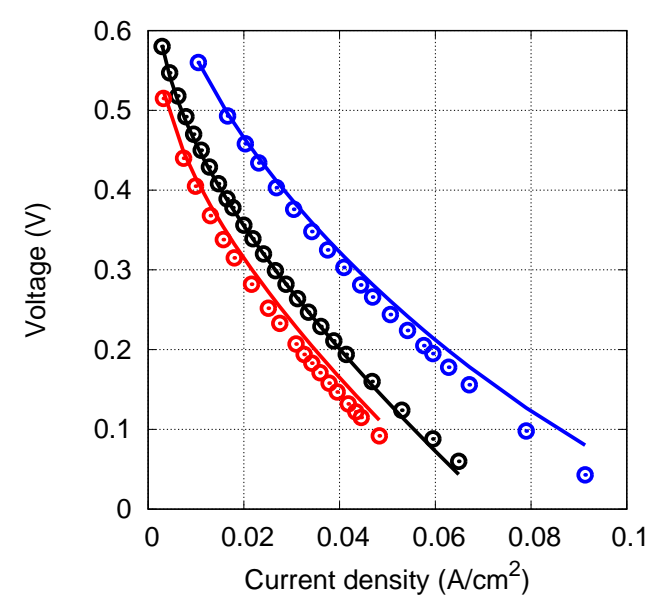

Figure 2: Comparisons between the experimental data and the predictions of semi-empirical model. (Line: Model; Dot: Experiment. Color is used to differentiate experimental cases.)

the deviation from model predications to experiments would increase as the operating voltage is approaching $0 \mathrm{~V}$. As those extreme operating conditions are not in the scope of present study, it has validated the feasibility and effectiveness of the proposed semi-empirical model.

\subsection{Adaptive operation strategy}

Large voltage deviation is frequently encountered in unoptimized DMFC operations (Fig. 3). It can not only worsen the performance of control system, but also increase the difficulty in the practical applications of DMFC systems. In practical use, the required output voltage $V_{\text {set }}$ and the range of operating current density $\left[j_{\min }, j_{\max }\right]$ are usually required by system designers. Taking advantage of the simplified semi-empirical model (as mentioned in Section 2.2), one can determine the output voltage from a specific current density $j$ and a series of operating parameters $\left[T, C_{M}, F_{M}, F_{A}\right]$, and the voltage deviation in a 


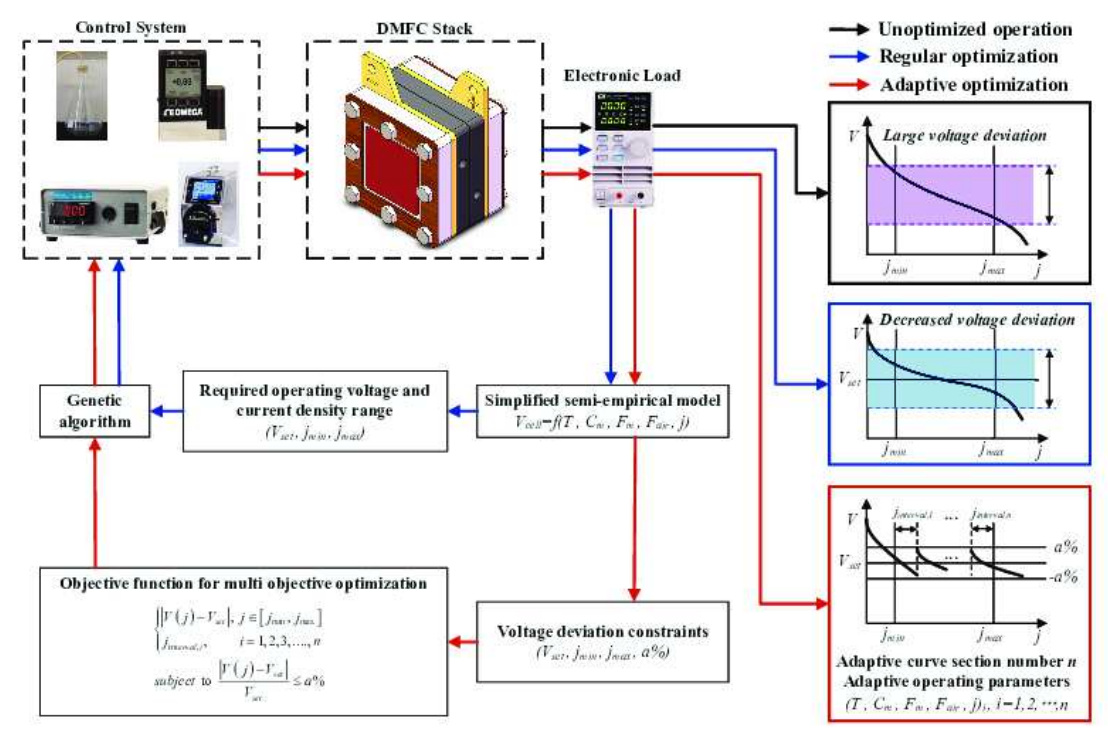

Figure 3: Schematic of operation strategies for DMFC systems.

required range of current density can be minimized using different optimization methods.

A regular optimization is usually on the basis of direct utilization of classical optimization method, such as genetic algorithm, to minimize the voltage deviation in the full range of required current density (Fig. 3). Compared with the unoptimized cases, a regular optimization could decrease the voltage deviation to a certain degree. However, a single I-V curve proposed by the regular optimization still can not guarantee a successful control of voltage stability in practical use, especially when the permitted voltage deviation $\pm a \%$ is required to be small.

An adaptive operation strategy is thus proposed. Its main idea is to adjust 
the operating parameters of a working DMFC system to fulfill the requirement of a small voltage deviation in a large range of working current density. Such a realtime adjustment brings out a switch of I-V curves, therefore, the performance of this modified DMFC operation need to be described by an integration of serval I-V curve sections.

With the objectives to decrease the voltage deviation $a \%$ and to enlarge the interval of current density $j_{\text {interval }}$, the adaptive operation strategy is designed to use the multi-objective optimization method [28] to freely adjusting the operating parameters to generate a required output voltage $V_{\text {set }}$ in the desired range of current density $\left[j_{\min }, j_{\max }\right]$. The objective function can be expressed as,

$$
\begin{aligned}
\min f\left(x, j_{\text {interval }}\right)= & w_{1}\left[V\left(j_{0}, x\right)-V\left(j_{0}+j_{\text {interal }}, x\right)\right]+w_{2} \frac{1}{j_{\text {interval }}}, \\
\text { s.t. } & \left\{\begin{array}{l}
\frac{\left|V\left(j_{0}, x\right)-V_{\text {set }}\right|}{V_{\text {set }}} \leq a \%, \\
\frac{\left|V\left(j_{0}+j_{\text {interval }}, x\right)-V_{\text {set }}\right|}{V_{\text {set }}} \leq a \%, \\
\text { for } \quad j \in\left[j_{\text {min }}, j_{\text {max }}\right] .
\end{array}\right.
\end{aligned}
$$

where $x$ denotes a random series of operating parameters (i.e., $\left[T, C_{M}, F_{M}, F_{A}\right]$ ), $w_{1}$ and $w_{2}$ are the weighting factors of the two objectives which are adaptively assigned to a specific DMFC system. Using the genetic algorithm, different sections of I-V curves, that correspond to different intervals of current density, can be searched and determined by varying the operating parameters $x$, and then they are connected to generate the integrated I-V curves. The switch from one I-V curve section to another can be fulfilled by the adjustment of operating 
parameters. As shown in Fig. 3. the optimization result is as an integration of more than one I-V curve sections, which ensures the desired voltage deviation in a required range of operating current density.

\subsection{Numerical model}

A three-dimensional numerical model was developed and validated to experimental results in our previous studies [22, 23]. It was designed by integrating the governing equations of continuity, momentum conservation, species transport and electrochemical phenomena. It has been successfully applied to investigate the energy conversion process in a DMFC system [23], which is just the same one as that we used in the present study. Therefore, this well-developed numerical model is adopted in the present study to investigate the underlying mechanisms of the adaptive operation strategy. For simplicity and concision, more details about the numerical model were not contained here, which can be available in Ref. [22, 23].

\section{Results and discussions}

\subsection{Large voltage variation in unoptimized operations}

Table 3: Operating parameters for testing cases with large voltage variations.

\begin{tabular}{ccccc}
\hline Case & $T(\mathrm{~K})$ & $C_{M}(\mathrm{M})$ & $F_{M}(\mathrm{ccm})$ & $F_{A}(\mathrm{ccm})$ \\
\hline 1 & 323 & 0.25 & 4 & 81.2 \\
2 & 323 & 0.50 & 5 & 140.8 \\
\hline
\end{tabular}

Large voltage variation was frequently encountered in our experiments. Two typical results of I-V curves are presented in Fig. 4 (top). They were observed 

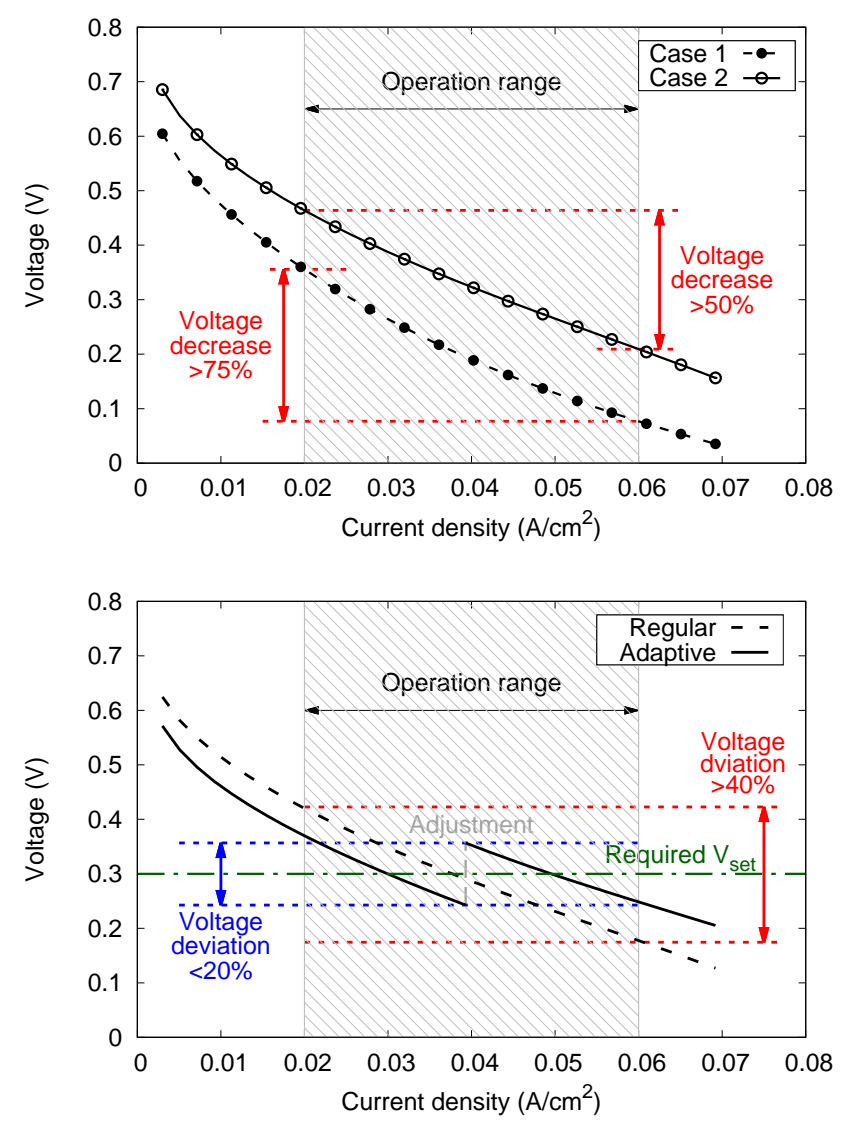

Figure 4: Large voltage variation in a constant range of current density (top) and the voltage stability enhancement using regular or adaptive optimization strategy (bottom).

in unoptimized experimental process, for which the operating conditions are summarized in Table 3 In a constant range of operating current density from 0.02 to $0.06 \mathrm{~A} / \mathrm{cm}^{2}$, the output voltage decreases from $0.3556 \mathrm{~V}$ to $0.0768 \mathrm{~V}$ for Case 1, and from $0.4638 \mathrm{~V}$ to $0.2092 \mathrm{~V}$ for Case 2. For both cases in Fig. 4. the increase of current density gives a significant discount in output voltage, which is even larger than $50 \%$. It indicates that the operational performance of the DMFC system can be significantly affected by the overpotential effect. 
Such a huge voltage variation is normally beyond the input limit of voltage stabilizers, the operation strategy is therefore in great need to minimize the voltage variation to a desired degree.

\subsection{Optimized operations}

The key point of operation strategy is to minimize the voltage deviation as the current density changes in a required range. Assuming that the current density varies between 0.02 and $0.06 \mathrm{~A} / \mathrm{cm}^{2}$ and the required voltage $V_{\text {set }}$ equals to $0.3 \mathrm{~V}$, the optimized operations using both regular and adaptive methods have been carefully examined. The operating condition that brings the smallest voltage deviation can be determined by the process of regular optimization using genetic algorithm, which is $T=322.8 \mathrm{~K}, C_{M}=0.6 \mathrm{M}, F_{M}=5.5 \mathrm{ccm}$ and $F_{A}=140.8 \mathrm{ccm}$. As shown in Fig. 4 (bottom), the voltage deviation was deduced to about $\pm 40 \%$ (-41.8\% to $40.93 \%$ ) by regular optimization. Although it is much better than those unoptimized cases, it is still far from the input requirement of regular voltage stabilizers. How to effectively reduce the voltage deviation is still significant for practical applications of fuel cell systems.

It is of interest to notice in Fig. 四 (bottom) that the voltage deviation can be obviously decreased to less than $20 \%$ using the adaptive operation strategy, with an operational adjustment imposed at the current density of $0.04 \mathrm{~A} / \mathrm{cm}^{2}$. Compared with the regular optimization that provides only one set of operating conditions, a real-time control of operating parameters by adaptive optimization can be an effective approach to make the voltage deviation be constrained in a desired range. The adaptive optimized I-V curve was composed by the gentle- 


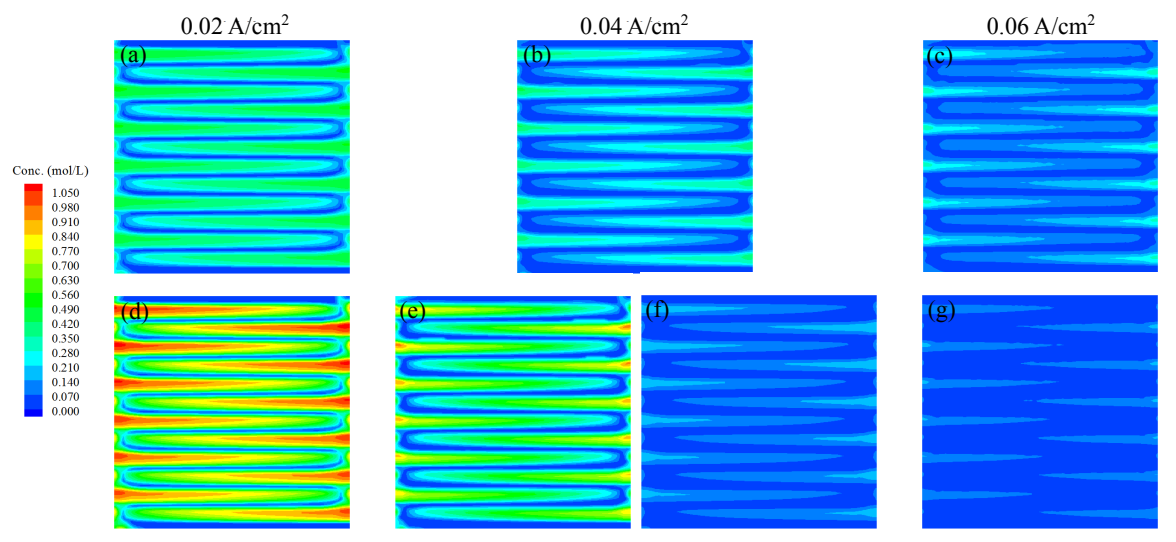

Figure 5: Distribution of methanol concentration on the interface plane of diffusion and catalyst layers. Top figures (a-c) represent the case with regular optimization, while the bottom ones $(\mathrm{d}-\mathrm{g})$ correspond to the case by adaptive optimization.

slope regions of two different $\mathrm{I}-\mathrm{V}$ curves, the left one is implemented at $T=$ $325.3 \mathrm{~K}, C_{M}=1.2 \mathrm{M}, F_{M}=5.4 \mathrm{ccm}$ and $F_{A}=140.6 \mathrm{ccm}$, while the right one is at $T=342.3 \mathrm{~K}, C_{M}=0.3 \mathrm{M}, F_{M}=5.5 \mathrm{ccm}$ and $F_{A}=140.7 \mathrm{ccm}$. It is obvious that a sharp voltage decrease can be largely subsided using the connected I-V curves, and this type of real-time control of operating parameters could be considered as "optimized flexible operations".

\subsection{Numerical analysis}

Numerical analysis was performed to answer the question "How does the adaptive optimization work on the enhancement of voltage stability in DMFCs?". Simulations were carried out using the proposed 3D numerical model [23]. The operating conditions were chosen to be the same as that for Fig. [4 (bottom) in Section 3.2 .

Fig. [5 shows the numerical results of methanol concentration distributing on the interface plane between the diffusion and catalyst layers, in which the top 

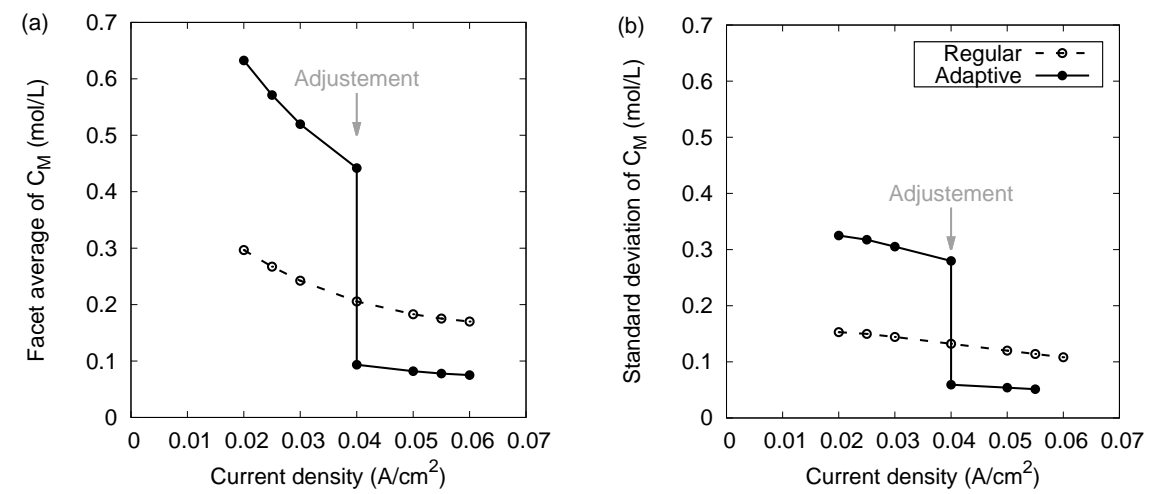

Figure 6: Facet average (a) and standard deviation (b) of methanol concentration on the interface plane between diffusion and catalyst layers.

figures (a-c) represent the case with regular optimization and the bottom ones (d-g) represent that by adaptive optimization. A remarkable characteristics is the stripped distributions of methanol concentrations, which is mainly caused by the parallel serpentine arrangement of flow channel in the DMFC system (more discussion is available in [21, 23, 27]). For the regular case, the overall color temperature of each figure becomes increasingly low as the current density increases from 0.02 to 0.04 and to $0.06 \mathrm{~A} / \mathrm{cm}^{2}$ (Fig. [ 5 (a-c)). It indicates that the methanol concentration decreases with the increase of current density, as the flux of methanol solution is constant for the given operating condition, this result agrees with the physical fact that a larger power generation is normally based on a higher fuel consumption. A similar result can be also found in the adaptive case. However, as the adaptive optimization imposes an adjustment of operating parameters, there are two figures $(e, f)$ at the current density $0.04 \mathrm{~A} / \mathrm{cm}^{2}$ indicating the change of methanol concentrations before and after the adjustment. It can be found that the adaptive adjustment has caused a 
significant decrease of methanol concentration, which results in a sharp increase of voltage at $0.04 \mathrm{~A} / \mathrm{cm}^{2}$ for the adaptive case in Fig. 4 (bottom). At a low current density $0.02 \mathrm{~A} / \mathrm{cm}^{2}$, the methanol concentration for the adaptive case (Fig. 5 (d)) is much higher than that with regular optimization (Fig. 5 (a)), but the situation reverses when a high current density $0.06 \mathrm{~A} / \mathrm{cm}^{2}$ is reached. Quantitative analysis about the methanol concentration distributing on the interface plane between the diffusion and catalyst layers is summarized in Fig. 6. As the current density increases from 0.02 to $0.06 \mathrm{~A} / \mathrm{cm}^{2}$, the facet average of methanol concentration gradually decreases from 0.3 to $0.17 \mathrm{~mol} / \mathrm{L}$ for regular case, while it declines sharply from 0.63 to $0.08 \mathrm{~mol} / \mathrm{L}$ for the adaptive one (Fig. 6 (a)). A slump occurring at $0.04 \mathrm{~A} / \mathrm{cm}^{2}$ for adaptive case is caused by the adjustment of operating conditions that has already been demonstrated in Fig. 4 (bottom) and 5. It is also of importance to notice that the slope of decrease for facet average of methanol concentration can be significantly modified by the adjustment. Compared to the regular case, it decreases much faster for the adaptive one before the adjustment, but a slower decrease can be achieved after the adjustment. It suggests that the adaptive optimization could effectively modulate the methanol consumption rate inside the DMFC system to improve the energy conversion process. The regulation effect of adaptive optimization can be also found in the result of standard deviation of methanol concentration (Fig. 6(b)) that can be regarded as a measurement of uniformity. It implies that the adjustment proposed by the adaptive optimization can markedly regulate the distribution of methanol concentration inside the DMFC system. 

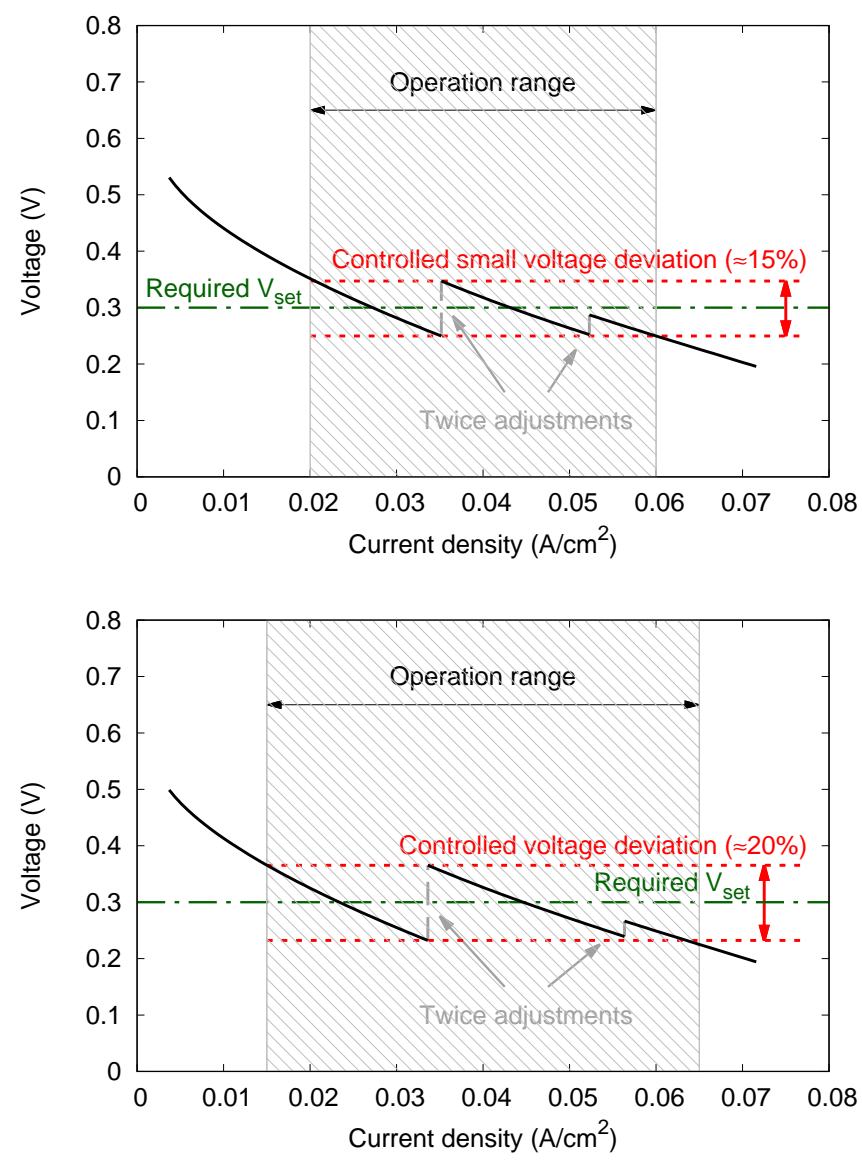

Figure 7: On-demand control of voltage stability using adaptive optimization strategy.

The above results have shown, in both qualitative and quantitative way, that the adjustment of operating parameters proposed by adaptive optimization is an effective way to regulate the methanol consumption inside a DMFC system. This is highly beneficial for the maintenance of voltage stability of the DMFC system that is required to work in a large range of current density. 


\subsection{On-demand adaptive operations}

Flexible operations are usually demanded by users in practical use, for example, decreasing the voltage deviation or extending the range of operating current density. Compared with the optimized operation in Fig. 4 (bottom) (Section 3.2) that is with a voltage deviation less than $20 \%$ in an operating current density range $[0.02,0.06] \mathrm{A} / \mathrm{cm}^{2}$, we consider a smaller voltage deviation or a larger operating current density in this section. Such a flexible requirement can be satisfied by on-demand control of operating parameters based on the adaptive optimization strategy.

Fig. 7 shows the optimized adaptive operations according to user's requirements. With a desired output voltage $0.3 \mathrm{~V}$, the voltage deviation was successfully decreased to about $\pm 15 \%$ in the first plot, while the operating current density was extended to $[0.015,0.065] \mathrm{A} / \mathrm{cm}^{2}$ in the second one. However, the cost to those high requirements is the number of adjustments. As shown in Fig. 7. one needs to implement twice adjustments to achieve a more stable voltage output in a larger working current density. Taking the first plot as example, the left I-V curve section in $[0.02,0.035] \mathrm{A} / \mathrm{cm}^{2}$ corresponds to the operation conditions of $T=314.7 \mathrm{~K}, C_{M}=1.1 \mathrm{M}, F_{M}=5.5 \mathrm{ccm}$ and $F_{A}=136.3 \mathrm{ccm}$, the middle one in $[0.035,0.052] \mathrm{A} / \mathrm{cm}^{2}$ corresponds to the operating conditions of $T=339.9 \mathrm{~K}, C_{M}=0.3 \mathrm{M}, F_{M}=5.5 \mathrm{ccm}$ and $F_{A}=126.8 \mathrm{ccm}$, and the right one in $[0.05,0.06] \mathrm{A} / \mathrm{cm}^{2}$ corresponds to the operating conditions of $T=342.8 \mathrm{~K}, C_{M}=0.3 \mathrm{M}, F_{M}=5.5 \mathrm{ccm}$ and $F_{A}=140.6 \mathrm{ccm}$. All of them are determined by the adaptive optimization strategy which ensures the smallest 
slope of each curve section in the corresponding interval of current density.

It is known to all that the DMFC system could provide a relatively stable voltage when it works at the "ohmic loss" range in one I-V curve. The adaptive optimization strategy provides us a method to search and to integrate pseudo "ohmic loss" sections in different I-V curves (which takes a small slope of voltage decrease as current density increases), to ensure the demanded voltage stability and to meet various requirements from DMFC users.

\subsection{Experimental validation}

Experimental work has also been carried out to study the effects of adaptive operation strategy on the voltage stability enhancement in practical use of DMFC systems. It was based on a customized DMFC stack that integrates a $5 \times 5 \mathrm{~cm}^{2}$ MEA with Nafion 115 membrane, for which Pt-Ru black $\left(4 \mathrm{mg} / \mathrm{cm}^{2}\right)$ and Pt-C $\left(0.3 \mathrm{mg} / \mathrm{cm}^{2}\right)$ were used as anode and cathode catalysts. The DMFC stack is required to work with an output voltage $0.38 \mathrm{~V}$, and the current density lies in the range of $[0.5,2.5] \mathrm{A} / \mathrm{cm}^{2}$. Fig. 8 (top) shows the comparisons of experimental I-V curves obtained with or without adaptive operation strategy. The voltage deviation was significantly decreased from $18.4 \%$ to $13.7 \%$ as the adaptive operation is implemented. It implies that the voltage stability can be effectively enhanced by the adaptive operation strategy.

Compared with the unoptimized case operating at a specific series of operation parameters of $T=338.0 \mathrm{~K}, C_{M}=0.5 \mathrm{M}, F_{M}=2.0 \mathrm{ccm}$ and $F_{A}=$ $1000.0 \mathrm{ccm}$, a manually adjustment of operating parameters should be imposed at at $1.4 \mathrm{~A} / \mathrm{cm}^{2}$ for adaptive operation. As system fluctuation and time-delay 

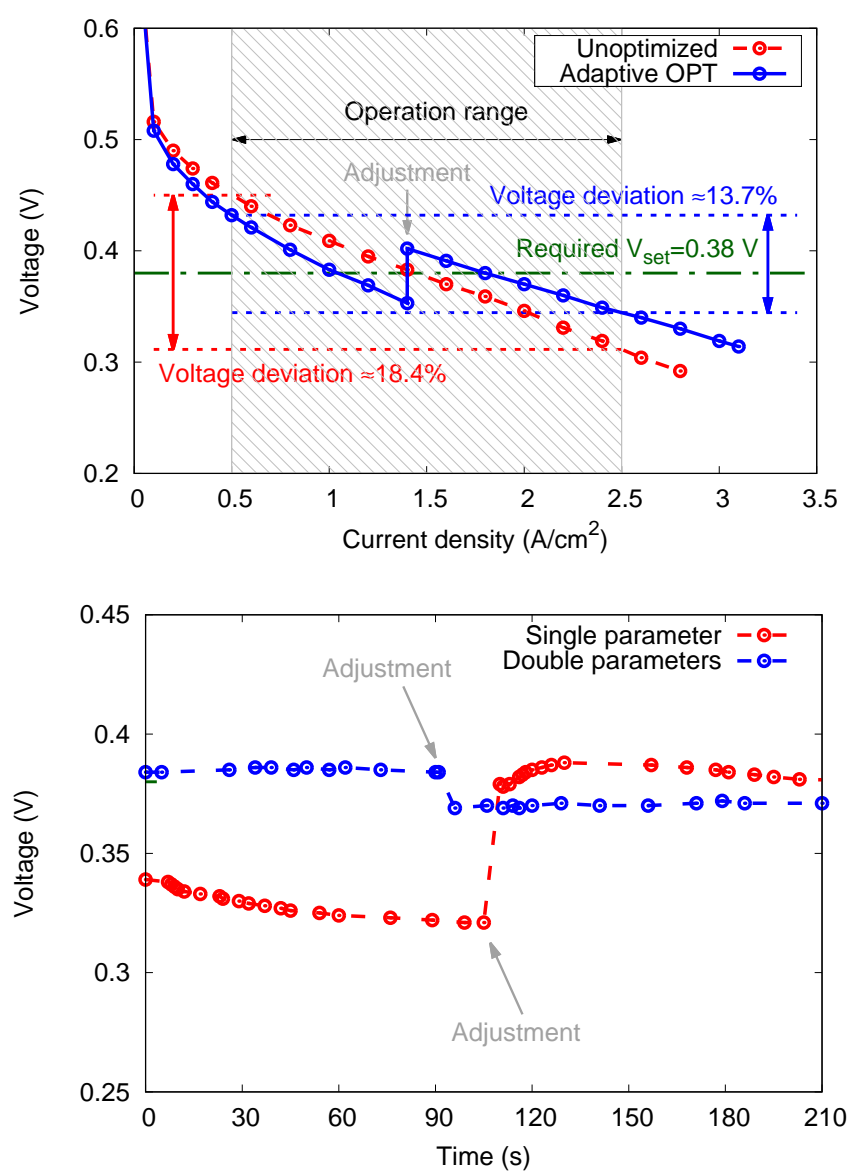

Figure 8: Experimental validation of adaptive optimized operations of a customized DMFC system (top) and its response to the adjustment of operating parameters (bottom).

would be introduced by manual operations, the system response to the manual adjustment of operation conditions need to be taken into account.

\subsection{System response to adjustments}

The adjustment of operating parameters can be regarded as a systematic disturbance to an operating DMFC. As such a disturbance could bring out negative effects to the system stability, the response of DMFC system to those 
operational adjustments is inevitable for the evaluation of the effectiveness of the adaptive operation strategy. Preliminary study about the system response was then carried out based on the customized DMFC stack (as described in Section 3.5). The change of output voltage, which can be regarded as a measurement of system stability, is recorded and analyzed as the operating parameters were adjusted.

Fig. 8 (bottom) shows the time evaluations of output voltage from a DMFC system that is modulated by the adjustment of one or two operation parameters. For the adjustment of single parameter, the input air flow rate $F_{A}$ was increased from 200 to $800 \mathrm{ccm}$, while the other operating parameters are kept at $T=$ $328.0 \mathrm{~K}, C_{M}=0.5 \mathrm{M}, F_{M}=3.0 \mathrm{ccm}$. Even if it would slightly change as time passes, the output voltage stays at about $0.34 \mathrm{~V}$, until the adjustment was imposed. A quick response took place quickly after the adjustment being imposed, as the output voltage was increased to about $0.38 \mathrm{~V}$ in 5 seconds. The same phenomenon can also be observed for the adjustment of double parameters, in which the input air flow rate $F_{A}$ was decreased from 500 to $100 \mathrm{ccm}$, the methanol solution flow rate $F_{M}$ was decreased from 3.0 to $1.0 \mathrm{ccm}$ and the other two operating parameters were kept at $T=328 \mathrm{~K}, C_{M}=0.5 \mathrm{M}$. It implies that the DMFC system can rapidly respond to the operational adjustment, no matter one or two operating parameters was changed. Such a rapid response of DMFC system to the change of operating parameters ensures the applicability of the proposed adaptive operation strategy, to some extent. However, compared to the input methanol concentration and the operating temperature, the flow 
rates of input air and the feed solution are relatively easy to be modulated with manual operations. A specific operating system for automatic adjustments can be highly beneficial for the implementation of adaptive operation strategy, which will be considered in our future work.

\section{Conclusions}

Concentrating on the problem of voltage instability, an adaptive operation strategy was successfully developed for on-demand control of DMFC systems to enhance the voltage stability in operating DMFC systems. The procedure of strategy developing has been discussed in details in the present study, and the feasibility and superiority of the proposed operation strategy have been systematically studied using both experimental and numerical techniques. The main findings in the present study can be summarized as follows,

1) Large voltage variation was frequently observed in the experiments using a typical DMFC stack. Although it can be decreased by a regular optimization method to a certain degree, it still needs improvements to avoid additional energy loss caused by the process of voltage stabilization. The adaptive optimization strategy can be a potential solution, as it could effectively decrease the voltage variation according to user's requirements by introducing certain operational adjustments.

2) On-demand DMFC operations, such as providing a smaller voltage deviation or a larger operating current density, was shown to be able to fulfilled by the adaptive optimization strategy. The adaptive optimization strategy provides a 
method to search and to integrate pseudo ohmic loss sections in different I-V curves to ensure the demanded voltage stability in flexible operations.

3) Numerical results were provided to study the underlying mechanisms of the adaptive optimization strategy, as the adjustment of operating parameters proposed by adaptive optimization strategy can be an effective way to regulate the methanol consumption inside the DMFC system. Experimental work was also carried out to validate the effects of adaptive operation strategy on the voltage stability in an operating DMFC system. A rapid response of DMFC system to the change of operating parameters (i.e. operational adjustment) was experimentally observed, which ensures the applicability of the proposed adaptive operation strategy.

\section{Acknowledgments}

Project supported by the National Natural Science Foundation of China (No. 11402084 \& 51505136), the Natural Science Foundation of Hunan Province (No. 2015JJ3051 \& 2017JJ3018), the self-determined project of State Key Laboratory of Advanced Design and Manufacturing for Vehicle Body (No. 51475002), the open fund of State Key Laboratory of Fluid Power \& Mechatronics Systems (No. GZKF-201615) and the Fundamental Research Funds for the Central Universities (Hunan University). 


\section{References}

[1] X. Luo, J. Wang, M. Dooner, J. Clarke, Overview of current development in electrical energy storage technologies and the application potential in power system operation, Applied Energy 137 (2015) 511-536.

[2] Y. Wang, K. Chen, J. Mishler, S. Cho, X. Adroher, A review of polymer electrolyte membrane fuel cells: technology, applications, and needs on fundamental research, Applied Energy 88 (4) (2011) 981-1007.

[3] M. Kamaruddin, S. Kamarudin, W. Daud, M. Masdar, An overview of fuel management in direct methanol fuel cells, Renewable and Sustainable Energy Reviews 24 (2013) 557-565.

[4] N. Karim, S. Kamarudin, An overview on non-platinum cathode catalysts for direct methanol fuel cell, Applied Energy 103 (2013) 212-220.

[5] P. Patel, M. Datta, P. Jampani, D. Hong, J. Poston, A. Manivannan, P. Kumta, High performance and durable nanostructured TiN supported $\mathrm{Pt}_{50}-\mathrm{Ru}_{50}$ anode catalyst for direct methanol fuel cell (DMFC), Journal of Power Sources 293 (2015) 437-446.

[6] T. Henriques, B. César, P. Branco, Increasing the efficiency of a portable pem fuel cell by altering the cathode channel geometry: a numerical and experimental study, Applied Energy 87 (4) (2010) 1400-1409.

[7] A. Ozden, M. Ercelik, D. Ouellette, C. Colpan, H. Ganjehsarabi, F. Hamdullahpur, Designing, modeling and performance investigation of bio- 
inspired flow field based DMFCs, International Journal of Hydrogen Energy (2017) 1-13.

[8] A. Mehmood, M. Scibioh, J. Prabhuram, M. An, H. Ha, A review on durability issues and restoration techniques in long-term operations of direct methanol fuel cells, Journal of Power Sources 297 (2015) 224-241.

[9] F. Barbir, PEM fuel cells: theory and practice, Academic Press, 2012.

[10] F. Zenith, U. Krewer, Modelling, dynamics and control of a portable DMFC system, Journal of Process Control 20 (5) (2010) 630-642.

[11] C.-Y. Chang, C.-H. Hsu, W.-J. Wang, C.-Y. Chen, The active control design for DMFC/Battery hybrid system using PIDNN, International Journal of Innovative Computing Information and Control 8 (3 B) (2012) 21012112.

[12] L. Fan, J. Zhang, C. Li, Model predictive control on constant voltage output of a proton exchange membrane fuel cell, Journal of Engineering Science and Technology Review 6 (2) (2013) 115-119.

[13] R. Keller, S. Ding, M. Müller, D. Stolten, Fault-tolerant model predictive control of a direct methanol-fuel cell system with actuator faults, Control Engineering Practice 66 (2017) 99-115.

[14] V. Oliveira, D. Falcao, C. Rangel, A. Pinto, Heat and mass transfer effects in a direct methanol fuel cell: A 1D model, International Journal of Hydrogen Energy 33 (14) (2008) 3818-3828. 
[15] J. Ko, P. Chippar, H. Ju, A one-dimensional, two-phase model for direct methanol fuel cells-Part I: Model development and parametric study, Energy 35 (5) (2010) 2149-2159.

[16] A. Kulikovsky, Two-dimensional numerical modelling of a direct methanol fuel cell, Journal of Applied Electrochemistry 30 (9) (2000) 1005-1014.

[17] E. Birgersson, J. Nordlund, H. Ekström, M. Vynnycky, G. Lindbergh, Reduced two-dimensional one-phase model for analysis of the anode of a DMFC, Journal of the Electrochemical Society 150 (10) (2003) A1368A1376.

[18] T. Yan, T.-C. Jen, Two-phase flow modeling of liquid-feed direct methanol fuel cell, International Journal of Heat and Mass Transfer 51 (5) (2008) $1192-1204$.

[19] N. Vasile, R. Doherty, A. Videla, S. Specchia, 3D multi-physics modeling of a gas diffusion electrode for oxygen reduction reaction for electrochemical energy conversion in PEM fuel cells, Applied Energy 175 (2016) 435-450.

[20] W. Yang, T. Zhao, C. Xu, Three-dimensional two-phase mass transport model for direct methanol fuel cells, Electrochimica Acta 53 (2) (2007) 853-862.

[21] B. Yu, Q. Yang, A. Kianimanesh, T. Freiheit, S. Park, H. Zhao, D. Xue, A CFD model with semi-empirical electrochemical relationships to study the influence of geometric and operating parameters on DMFC performance, International Journal of Hydrogen Energy 38 (23) (2013) 9873-9885. 
[22] Q. Yang, A. Kianimanesh, T. Freiheit, S. Park, D. Xue, A semi-empirical model considering the influence of operating parameters on performance for a direct methanol fuel cell, Journal of Power Sources 196 (24) (2011) 10640-10651.

[23] X. Hu, X. Wang, J. Chen, Q. Yang, D. Jin, X. Qiu, Numerical investigations of the combined effects of flow rate and methanol concentration on DMFC performance, Energies 10 (8) (2017) 1094.

[24] D. Chu, R. Jiang, Effect of operating conditions on energy efficiency for a small passive direct methanol fuel cell, Electrochimica Acta 51 (26) (2006) $5829-5835$.

[25] V. Silva, A. Rouboa, Optimizing the DMFC operating conditions using a response surface method, Applied Mathematics and Computation 218 (12) (2012) 6733-6743.

[26] K.-T. Fang, D. Lin, P. Winker, Y. Zhang, Uniform design: theory and application, Technometrics 42 (3) (2000) 237-248.

[27] A. Kianimanesh, Q. Yang, S. Park, D. Xue, T. Freiheit, Model for the degradation performance of a single-cell direct methanol fuel cell under varying operational conditions, Fuel Cells 13 (6) (2013) 1005-1017.

[28] H. Ren, W. Zhou, K. Nakagami, W. Gao, Q. Wu, Multi-objective optimization for the operation of distributed energy systems considering economic and environmental aspects, Applied Energy 87 (12) (2010) 3642-3651. 